TRANSACTIONS OF THE

AMERICAN MATHEMATICAL SOCIETY

Volume 365, Number 11, November 2013, Pages 5737-5758

S 0002-9947(2013)05703-0

Article electronically published on April 25, 2013

\title{
RECOVERY OF A SOURCE TERM OR A SPEED WITH ONE MEASUREMENT AND APPLICATIONS
}

\author{
PLAMEN STEFANOV AND GUNTHER UHLMANN
}

\begin{abstract}
We study the problem of recovery of the source $a(t, x) F(x)$ in the wave equation in anisotropic medium with $a$ known so that $a(0, x) \neq 0$, with a single measurement. We use Carleman estimates combined with geometric arguments and give sharp conditions for uniqueness. We also study the nonlinear problem of recovery of the sound speed in the equation $u_{t t}-c^{2}(x) \Delta u=0$ with one measurement. We give sharp conditions for stability as well. An application to thermoacoustic tomography is also presented.
\end{abstract}

\section{INTRODUCTION}

The purpose of this paper is to give sharp conditions for the recovery of a source term in the wave equation in anisotropic media modeled by a Riemannian metric by a single boundary measurement. In the process, we give a more geometric treatment of the problem. This linear problem appears as a linearization and actually, as the full non-linear version, of the problem of recovery of a sound speed, given the source. It has applications to thermoacoustic tomography. We are also inspired by the related works [7, 8, 9, [11, Theorem 8.2.2]. The method in these papers uses Carleman estimates for hyperbolic inverse problems that originate in the work [2, where the case of the wave equation with a potential with non-zero initial data is considered.

The main problem that we have in mind is the following. Let $u$ solve

$$
\left\{\begin{aligned}
\left(\partial_{t}^{2}-c^{2} \Delta\right) u & =0 \quad \text { in }(0, T) \times \mathbf{R}^{n}, \\
\left.u\right|_{t=0} & =f, \\
\left.\partial_{t} u\right|_{t=0} & =0
\end{aligned}\right.
$$

where $c=c(x)>0$, and $T>0$ is fixed. Let $c=1$ outside some domain $\Omega$ with a smooth strictly convex boundary. Given $f$, and $u$ restricted to $[0, T] \times \partial \Omega$, is it possible to reconstruct the speed $c$ ? Ideally, we want to do this with data on a part of $\partial \Omega$ as well. Next, assuming that we can, how stable is this? Clearly, some conditions on $f$ are needed since when $f=0$, for example, we get no information about $c$. This inverse problem is clearly non-linear.

Received by the editors March 13, 2011 and, in revised form, August 10, 2011.

2010 Mathematics Subject Classification. Primary 35L05, 35R30.

The first author was partially supported by an NSF Grant DMS-0800428 and a Simons Visiting Professorship.

The second author was partially supported by an NSF, a Senior Clay Award and Chancellor Professorship at the University of California, Berkeley. 
If we have two speeds $c$ and $\tilde{c}$, then $w=\tilde{u}-u$ solves

$$
\left\{\begin{aligned}
\left(\partial_{t}^{2}-c^{2} \Delta\right) w & =a(t, x) F(x) \quad \text { in }(0, T) \times \mathbf{R}^{n}, \\
\left.w\right|_{t=0} & =0 \\
\left.\partial_{t} w\right|_{t=0} & =0
\end{aligned}\right.
$$

with

$$
F:=\tilde{c}^{2}-c^{2}, \quad a=\Delta \tilde{u} .
$$

We consider the more general linear problem of recovery of a function $F$, given $a$, and $w$ restricted to $[0, T] \times \partial \Omega$, or on a part of it. Again, some condition on $a$ is needed since when $a=0$, for example, $F$ cannot be recovered. We actually replace $c^{2} \Delta$ in (2) by the Laplace-Beltrami operator $\Delta_{g}$ related to some metric, plus lower order terms.

Similar problems but for the recovery of a potential or the term $p(x)$ in $\nabla \cdot p \nabla$ are studied in [17, 7, 8, 9]. A general abstract theorem of this type is presented in [11, Theorem 8.2.2]. In [9] and [11, the principal part of the wave equation has variable coefficients, thus the geometry is non-Euclidean. This requires some assumptions on the speed or the metric. The method of the proof is to use Carleman estimates, and the assumptions are needed to satisfy the pseudo-convexity condition. Those assumptions are not sharp however. In fact, the proofs are essentially "Euclidean", and roughly speaking, the conditions on the speed or on the metric require that the proof still works under an Euclidean treatment. Also, one global pseudo-convex function is used. One of the goals of this work is to formulate such conditions in a geometric way and, in particular, to obtain a sharper one, thus extending the results to a larger class of speeds/metrics, and also prove local results.

There are many works on related problems, including boundary control, for example, 1, 15, 30, 29. There the conditions on the metric are more geometric, requiring existence of a global convex function, or a somewhat general condition of existence of a global vector field with certain properties. The proofs are still based on Carleman estimates, but the goal is to recover non-trivial initial conditions, assuming, say, a Neumann boundary condition, and measuring Dirichlet data on a part of $\partial \Omega$. The conditions on $T$ are formulated in terms of lower bounds of the speed and are not sharp. The geometry of the rays in those problems however is different from the application that we have in mind - there are reflections at the boundary. On the other hand, the methods there could probably be adapted to the problems studied in the works that we cited above.

The pseudo-convexity condition needed for the Carleman estimates that we use is satisfied by assuming that the region where we prove unique continuation is foliated by a continuous family $\Sigma_{s}$ of strictly convex surfaces. In the case of data on a part $\Gamma$ of $\partial \Omega$, we require those surfaces to intersect $\partial \Omega$ in $\Gamma$, which can be viewed as propagation of uniqueness from $\partial \Omega$ to the interior along strictly convex surfaces. In contrast to the other works in this direction, we are not trying to construct one strongly pseudo-convex function. Instead, we prove unique continuation by incremental steps, each time using a different strongly pseudo-convex function.

We now describe the results in the paper. We start in section 2 with the uniqueness Theorem [2.1, that is a version of [11, Theorem 8.2.2]. The time interval is $(-T, T)$, there is no initial condition for $w_{t}$ at $t=0$, and we study the problem of unique recovery of $F$ in (2) given Cauchy data on a part of $(-T, T) \times \partial \Omega$. We view this theorem more as a tool than a goal, and the requirement that the time interval 
is symmetric about $t=0$ will be satisfied later by studying problems with solutions that have even extensions in $t$, like (1). In the rest of that section we show two ways to satisfy the convexity requirement. First, assuming $\partial \Omega$ is strictly convex, we show in Theorem 2.2 that $F=0$ in some collar neighborhood of $\partial \Omega$ of the kind $\operatorname{dist}(x, \partial \Omega) \leq T \ll 1$, as long as the surfaces $\operatorname{dist}(x, \partial \Omega)=s, s \in[0, T]$ are smooth and still strictly convex. The second one is to show that $F=0$ in a subset of $\Omega$ that can be foliated by strictly convex surfaces starting from ones outside $\Omega$; see Theorem 2.3. This only requires Cauchy data on a part of $\partial \Omega$, where those surfaces intersect $\partial \Omega$, and proves $F=0$ in a subdomain. The condition on $T$ is sharp. We give a few examples.

In section 3 we study the non-linear problem of recovery of the speed $c$ in (1) and the linear one of recovery of the source $F$ in (2) described above. The time interval is $(0, T)$ now, but the initial condition $u_{t}=0$ for $t=0$ in (11), and the requirement that $a$ in (2) has a sufficiently regular even extension in the $t$ variable, allow us to use the results in the previous section. In contrast to the boundary control problems, in the thermoacoustic problem we are given the Dirichlet data on $(0, T) \times \partial \Omega$ or on a part of it, but no Neumann data. On the other hand, we know that the solution extends for $x \notin \Omega$ as a solution again, and the initial data at $t=0$ is zero there. This allows us, in Lemma 3.1, to recover the Neumann data from the Dirichlet one in case of data on the whole $\partial \Omega$. Then we extend the solution in an even way for $t<0$ and apply the results in section 2, The main requirement is for $\Omega$ to have a foliation of strictly convex surfaces and the time interval $(0, T)$ to be sharp.

The partial data case, with observations on $(0, T) \times \Gamma$, where $\Gamma \subset \partial \Omega$ in the thermoacoustic setting, is studied in Theorem 3.2. The main difficulty is the need to recover the Neumann data there as well. Then one directly applies the results in section 2. We show that one can recover $F$, respectively $c$, in some neighborhood of $\Gamma$ that might be smaller compared to the case of having Cauchy data on the whole $(0, T) \times \Gamma$. There is a new "cone" condition that might shrink the domain where we prove $F=0$, or respectively $\tilde{c}=c$.

At the end of section 3. we study the stability of the linear and non-linear problems for (11) and (2), respectively. As a general principle, for stability, we need to be able to detect all singularities; see (51). For the linear problem at least, this is a necessary and sufficient condition for stability in any Sobolev spaces; see [21. The corresponding stability estimates are formulated in Theorems 3.4 and 3.5. Lipschitz stability estimates for related problems were proven earlier in [17, 7, 8].

\section{A Uniqueness ReSUlt FOR RECOVERY OF A SOURCE WITH ONE MEASUREMENT}

Let $\Omega$ be a bounded domain in $\mathbf{R}^{n}$ with a smooth oriented boundary and let

$$
P=\partial_{t}^{2}-\Delta_{g}+\sum_{j} b_{j} \partial_{x^{j}}+c
$$

be a differential operator in $Q:=(-T, T) \times \Omega \subset \mathbf{R}^{n}$, where $g$ is a smooth Riemannian metric on $\Omega$, and $a_{j}, b, c$ are smooth functions on $\bar{Q}$.

The level surface $\Sigma=\{\psi=0\}$ of some smooth function $\psi$ is called strongly pseudo-convex w.r.t. the hyperbolic operator $P$, if

(i) $\Sigma$ is non-characteristic, i.e., $\left|\psi_{t}\right| \neq\left|\mathrm{d}_{x} \psi\right|$ when $\psi=0$, and 
(ii) $H_{p}^{2} \psi>0$ on $T^{*} \bar{\Omega} \backslash 0$ whenever $\psi=p=H_{p} \psi=0$,

where $H_{p}$ is the Hamiltonian vector field of the principal symbol $p=-\lambda^{2}+|\xi|^{2}$ of $P$, and $\lambda$ is the dual variable to $t$; see, e.g., [27]. Here and below, $|\cdot|$ is the norm in the metric $g$ of a covector or a vector. The second condition says that $\Sigma$ is strictly convex w.r.t. the null bicharacteristics of $p$, when viewed from $\psi>0$. In other words, the tangent null bicharacteristics to $\Sigma$ are curved towards $\psi>0$.

The function $\phi$ with a non-degenerate differential and non-characteristic level set $\phi=0$ is pseudo-convex if a condition stronger than (ii) is satisfied. We are not going to formulate that condition; it would be enough for our purposes to use the well-known fact that if $\Sigma=\{\psi=0\}$ as above is pseudo-convex, then for $\mu \gg 1, \phi=\exp (\mu \psi)-1$ is a pseudo-convex function, non-degenerate on $\Sigma$, and $\Sigma=\{\phi=0\}$; moreover, $\{\phi>0\}=\{\psi>0\}$. For details, we refer the reader to [27].

Let $\phi$ be strongly pseudo-convex in $\bar{Q}$ w.r.t. $P$. Then it is well known that for all $u \in C_{0}^{2}(Q)$,

$$
\tau \int e^{2 \tau \phi}\left(\left|u_{t}\right|^{2}+|\nabla u|^{2}+\tau^{2}|u|^{2}\right) \mathrm{d} t \mathrm{~d} x \leq C \int e^{2 \tau \phi}|P u|^{2} \mathrm{~d} t \mathrm{~d} x, \quad \tau>1 ;
$$

see [27, 11, 10].

To reformulate condition (ii) in the tangent bundle, recall that the metric $g$ provides a natural isomorphism between covectors and vectors by the formula $\Phi$ : $(x, \xi) \mapsto(x, v)$, where $\xi_{i}=g_{i j}(x) v^{j}$ in local coordinates, where $v$ is a vector at $x$. For any function $\psi$ on $T^{*} \Omega$, one gets a function $\Phi^{*} \psi$ on $T \Omega$. Let $q=|\xi|^{2} / 2$ be the " $x$ part" of $p$, rescaled for convenience. It is known that $H_{q}=\Phi_{*} G \Phi^{*}$, where $G$ is the generator of the geodesic flow. Also, the energy level $q=1 / 2$ is pushed forward to the unit sphere bundle $S \Omega$.

We have $H_{p / 2}=-\lambda \partial_{t}+H_{q}$, therefore,

$$
\frac{1}{4} H_{p}^{2} \psi=\left(\lambda \partial_{t}-H_{q}\right)^{2} \psi
$$

We identify covectors with vectors by the map $\Phi$, to get that condition (ii) in $Q$ is equivalent to

$$
\psi=0, \quad \psi_{t}-G \psi=0 \quad \Longrightarrow \quad\left(\partial_{t}-G\right)^{2} \psi>0 \quad \text { for }(t, x) \in Q,|\xi|=1,
$$

and we use the fact that $p=0$ implies $\lambda^{2}=|\xi|^{2}$ as well as the homogeneity properties of $G$ w.r.t. $\xi$.

Let us look for $\psi$ of the type

$$
\psi=r^{2}(x)-\delta t^{2}-p, \quad 0<\delta<1,
$$

with $p$ a parameter. Then, to satisfy (ii), it is enough to have

$$
G^{2}\left(r^{2} / 2\right)>\delta|\xi|^{2} \text {. }
$$

Since we eventually want to take the limit $\delta \rightarrow 1$ to get sharp results, we arrive at the condition

$$
G^{2}\left(r^{2} / 2\right) \geq|\xi|^{2}
$$

It is enough to have this inequality in the $x$-projection of $\Sigma$ in $\bar{\Omega}$, as the first condition in (6) indicates. Note that this guarantees (ii) only; we still have to choose $r$ so that (i) holds. The latter is equivalent to

$$
\left|\mathrm{d}\left(r^{2} / 2\right)\right| \neq \delta|t| \quad \text { on } \Sigma \cap \bar{Q} \text {. }
$$


Example 1. Let $r(x)=\left|x-x_{0}\right|$ in $\mathbf{R}^{n}$ with some fixed $x_{0}$, then $\psi=\left|x-x_{0}\right|^{2}-$ $\delta t^{2}-p$. Then

$$
G^{2}\left(r^{2} / 2\right)=\left(\xi \cdot \nabla_{x}\right)^{2}\left|x-x_{0}\right|^{2} / 2=|\xi|^{2},
$$

and (8) holds. Condition (9) is satisfied for $p>0$ because $\left|\mathrm{d}\left(r^{2} / 2\right)\right|^{2}=\left|x-x_{0}\right|^{2}=$ $\delta t^{2}+p>\delta^{2} t^{2}$. Then $\Sigma$ is a hyperboloid of one sheet.

More generally, let $r(x)=\rho\left(x, x_{0}\right)$, where $\rho$ is the distance in the metric $g$. This is the function that has been used in the Riemannian case. It satisfies (7) for $r \ll 1$ only, in general. For metrics of negative curvature, there is no restriction; see [19].

In this paper, $\partial / \partial \nu$ denotes the exterior normal derivative to $\partial \Omega$.

Theorem 2.1. Let $Q=(-T, T) \times \Omega$, and

$$
\partial_{t}^{j} a \in C(\bar{Q}), j \leq 2, F \in L^{2}(\Omega) \text {, and } \partial_{t}^{j} \partial_{x}^{\alpha} u \in L^{2}(Q), j \leq 3,|\alpha| \leq 1 .
$$

Let $u$ be a (non-unique) solution of

$$
\left\{\begin{aligned}
P u & =a(t, x) F(x) & & \text { in }(-T, T) \times \Omega, \\
\left.u\right|_{t=0} & =0 & & \text { in } \Omega .
\end{aligned}\right.
$$

Let $\phi$ be a strongly pseudo-convex function in $\bar{Q}$. Let $\mathcal{G} \subset(-T, T) \times \partial \Omega$ be an open set with

$$
\phi<0 \text { on } \partial Q \backslash \mathcal{G}, \quad \text { and } \phi(t, \cdot) \leq \phi(0, \cdot) \text { for }|t|<T .
$$

Let $\operatorname{supp} F \subset K$, where $K \subset \bar{\Omega}$ is compact, and

$$
a(0, x) \neq 0, \quad \forall x \in K .
$$

If

$$
u=\partial u / \partial \nu=0 \quad \text { on } \mathcal{G},
$$

then

$$
F=0 \quad \text { in }\{x \in \Omega ; \phi(0, x)>0\} .
$$

Proof. We follow the proof of [11, Theorem 8.2.2]. Set $Q_{\varepsilon}=Q \cap\{\phi>\varepsilon\}$. Fix $\varepsilon>0$, let $\chi \in C^{\infty}$ be such that $\chi=1$ in $Q_{\varepsilon}$, and supp $\chi \subset \bar{Q}_{0}$. We will apply the Carleman estimate (5) to $\partial_{t}^{j} \chi u, j=0$ and $j=2$, by shrinking $Q_{0}$ to $Q_{\varepsilon}$ on the left. Here we are using the fact that $u$ has zero Cauchy data on $\mathcal{G}$. Then $\partial_{t}^{j} \chi u$ can be approximated by $C_{0}^{\infty}(Q)$ functions in the $H^{2}$ norms; see also Lemma 2.1. We have

$$
P \partial_{t}^{j} \chi u=\partial_{t}^{j}(\chi P u+[P, \chi] u)=\partial_{t}^{j}(\chi a F+[P, \chi] u),
$$

where the commutator $[P, \chi]$ is a differential operator of order 1 . Since $\chi=1$ on $Q_{\varepsilon}$, we get

$$
\begin{aligned}
\tau \int_{Q_{\varepsilon}} & e^{2 \tau \phi}\left(\tau^{2}|u|^{2}+\tau^{2}\left|u_{t t}\right|^{2}+\left|u_{t t t}\right|^{2}\right) \mathrm{d} \sigma \\
\leq & C\left(\int_{Q} e^{2 \tau \phi}|F|^{2} \mathrm{~d} \sigma+\int_{Q \backslash Q_{\varepsilon}} e^{2 \tau \phi} \sum_{j=0}^{2} \sum_{|\alpha| \leq 1}\left|\partial_{t}^{j} \partial_{t, x}^{\alpha} u\right|^{2} \mathrm{~d} \sigma\right) \\
\leq & C \int_{Q} e^{2 \tau \phi}|F|^{2} \mathrm{~d} \sigma+C e^{2 \tau \varepsilon},
\end{aligned}
$$


where $\mathrm{d} \sigma=\mathrm{d} t \mathrm{~d} \operatorname{Vol}(x)$ is the natural measure on $Q$. We will estimate the first term on the right-hand side above. From equation (11) and its initial condition, $u_{t t}(0, \cdot)=a(0, \cdot) F$. By (13) $),|F| \leq C\left|u_{t t}(0, \cdot)\right|$. Using (12), we get

$$
\int_{Q} e^{2 \tau \phi}|F|^{2} \mathrm{~d} \sigma \leq 2 C^{2} T \int_{\Omega} e^{2 \tau \phi(0, \cdot)}\left|u_{t t}(0, \cdot)\right|^{2} \mathrm{~d} \operatorname{Vol}(x) .
$$

This integral admits the estimate

$$
\begin{aligned}
\int_{\Omega} e^{2 \tau \phi(0, \cdot)}\left|u_{t t}(0, \cdot)\right|^{2} \mathrm{~d} \operatorname{Vol}(x)= & -\int_{0}^{T} \int_{\Omega} \frac{\partial}{\partial s}\left(e^{2 \tau \phi(s, \cdot)}\left|u_{t t}(s, \cdot)\right|^{2}\right) \mathrm{d} \operatorname{Vol}(x) \mathrm{d} s \\
& +\int_{\Omega} e^{2 \tau \phi(T, \cdot)}\left|u_{t t}(T, \cdot)\right|^{2} \mathrm{~d} \operatorname{Vol}(x) \\
\leq & C \int_{Q} e^{2 \tau \phi}\left(\tau\left|u_{t t}\right|^{2}+\tau^{-1}\left|u_{t t t}\right|^{2}\right) \mathrm{d} \sigma+C,
\end{aligned}
$$

because $\phi(T, \cdot) \leq 0$, and by the Cauchy inequality. This inequality, together with (16), estimate the integral of the first term on the right-hand side of (15). Therefore,

$$
\begin{aligned}
& \tau \int_{Q_{\varepsilon}} e^{2 \tau \phi}\left(\tau^{2}|u|^{2}+\tau^{2}\left|u_{t t}\right|^{2}+\left|u_{t t t}\right|^{2}\right) \mathrm{d} \sigma \\
& \quad \leq C\left(\tau^{-1} \int_{Q} e^{2 \tau \phi}\left(\tau^{2}\left|u_{t t}\right|^{2}+\left|u_{t t t}\right|^{2}\right) \mathrm{d} \sigma+e^{2 \tau \varepsilon}\right) .
\end{aligned}
$$

Split the integration on the right into $Q_{\varepsilon}$ and $Q \backslash Q_{\varepsilon}$. For $\tau \gg 1$, the integral over $Q_{\varepsilon}$ will be absorbed by the left-hand side. On $Q \backslash Q_{\varepsilon}$, we have $e^{2 \tau \phi} \leq e^{2 \tau \varepsilon}$. Therefore,

$$
\tau \int_{Q_{\varepsilon}} e^{2 \tau \phi}|u|^{2} \mathrm{~d} \sigma \leq C e^{2 \tau \varepsilon}, \quad \text { for } \tau \gg 1
$$

Thus,

$$
\int_{Q_{\varepsilon}} e^{2 \tau(\phi-\varepsilon)}|u|^{2} \mathrm{~d} \sigma \leq C / \tau, \quad \text { for } \tau \gg 1
$$

Since $\phi-\varepsilon \geq 0$ in $Q_{\varepsilon}$, letting $\tau \rightarrow \infty$, we get $u=0$ in $Q_{\varepsilon}$. Since $\varepsilon>0$ is arbitrary, we get $u=0$ in $Q_{0}$. By (11), $a F=0$ in $Q_{0}$, and in particular, $a(0, \cdot) F=0$ in $Q_{0} \cap\{t=0\}=\{x \in \Omega ; \phi(0, x)>0\}$. By the ellipticity condition (13), $F=0$ there as well. This proves the theorem.

The following lemma will allow us to apply the proof of the theorem to a larger class of non-smooth boundaries.

Lemma 2.1. Let $D \subset \mathbf{R}^{n}$ be open, and assume that near each point $x_{0} \in \partial D$, $D$ is represented by $y>0$ and $z>0$, where $y$ and $z$ are functions with non-zero differentials satisfying the following condition:

$$
\text { if }\{y=0\} \text { and }\{z=0\} \text { intersect, then }\{y=0\} \backslash\{z=0\} \text { is dense in }\{y=0\} \text {. }
$$

Let $u \in C^{2}(\bar{D})$ have Cauchy data 0 on $\partial D$ in the sense that extended as 0 outside $D$, it still belongs to $C^{2}$. Then $u$ can be approximated by $C_{0}^{2}(D)$ functions in $H^{2}\left(\mathbf{R}^{n}\right)$.

Proof. Let $\chi \in C_{0}^{\infty}(\mathbf{R})$ be such that $\chi=1$ near 0 . Set locally, near $x_{0} \in \partial D$,

$$
u_{\varepsilon}=(1-\chi(y / \varepsilon))(1-\chi(z / \varepsilon)) u .
$$


Using a partition of unity, we define such $u_{\varepsilon} \in C_{0}^{2}(D)$. We claim that $u_{\varepsilon} \rightarrow u$ in $H^{2}\left(\mathbf{R}^{n}\right)$, as $\varepsilon \rightarrow 0+$. Take the second derivatives of $u-u_{\varepsilon}$ to see that we need to show that the following terms converge to 0 in $L^{2}\left(\mathbf{R}^{n}\right)$ :

$$
\varepsilon^{-1} \chi^{\prime}(y / \varepsilon) u, \quad \varepsilon^{-2} \chi^{\prime \prime}(y / \varepsilon) u, \quad \varepsilon^{-2} \chi^{\prime}(y / \varepsilon) \chi^{\prime}(z / \varepsilon) u,
$$

where we used the fact that the derivatives of $y$ and $z$ are bounded. We do not list terms involving lower powers of $\varepsilon^{-1}$ and derivatives of $u$, for which analysis is similar. Similarly, we will not analyze the zero and the first order derivatives of $u_{\varepsilon}$. Since $y / \varepsilon$ and $z / \varepsilon$ are bounded on the support of $\chi^{\prime}(y / \varepsilon)$ and $\chi^{\prime}(z / \varepsilon)$, respectively, we may replace the leading coefficient $\varepsilon^{-1}$ in the first term by $y^{-1}$, etc. Since $u=0$ for $z=0$, and $\mathrm{d} z \neq 0$, we have $u=z u_{1}$, where $u_{1} \in C^{1}$. Next, $u_{1}=0$ for $y=0$ at least when $z \neq 0$. That set of $y$ 's however is dense in $\{y=0\}$ by assumption. By continuity, $u_{1}=0$ for $y=0$. Thus $u_{1}=y u_{2}$ with $u_{2} \in C^{0}$, therefore, $u=z y u_{2}$. Now, the proof of the lemma follows from the fact that $\chi^{\prime \prime}(y / \varepsilon)$ tends to 0 in $L^{2}\left(\mathbf{R}^{n}\right)$ and this is also true if we replace $\chi^{\prime \prime}$ by $\chi^{\prime}$ or $\chi$.

Next we recall a unique continuation result due to Tataru [26]; see also 23, Theorem 4]. Assume that a locally $H^{1}$ function $u$ solves the homogeneous wave equation $P u=0$ (near the set indicated in (17) below) and vanishes in an open set containing $(-T, T) \times\left\{x_{0}\right\}$ for some $x_{0}$ and $T>0$. Then

$$
u(t, x)=0 \quad \text { for } \quad|t|+\operatorname{dist}\left(x_{0}, x\right)<T .
$$

Based on that, one can show unique continuation of Cauchy data on $\mathbf{R} \times \partial \Omega$ to their domain of influence; see, e.g., [12, Theorem 3.16].

Proposition 2.1. Let $u \in H^{1}$ solve the homogeneous wave equation $P u=0$ in $[-T, T] \times \Omega$. Assume that $u$ has Cauchy data zero on $[-T, T] \times \Gamma$, where $\Gamma \subset \partial \Omega$ is open. Then $u=0$ in the domain of influence $\{(t, x) \in[-T, T] \times \Omega ; \operatorname{dist}(x, \Gamma) \leq$ $T-|t|\}$.

The next theorem in fact follows from Theorem 2.3 below, but its proof is simpler, and it serves as a basis for the proof of Theorem 2.3. We refer to Figure 1 for an illustration. Recall that in a Riemannian manifold, the (hyper) surface $S$ with a chosen local orientation given by a smooth normal unit vector field $\nu$ is called strictly convex at $x \in S$ if the second fundamental form $\operatorname{II}(v, w)=\left\langle\nabla_{v} \nu, w\right\rangle$ is positive at $x$; see, e.g., [20, p. 112]. Since $\partial \Omega$ is oriented, it has a fixed choice of $\nu(x)$.

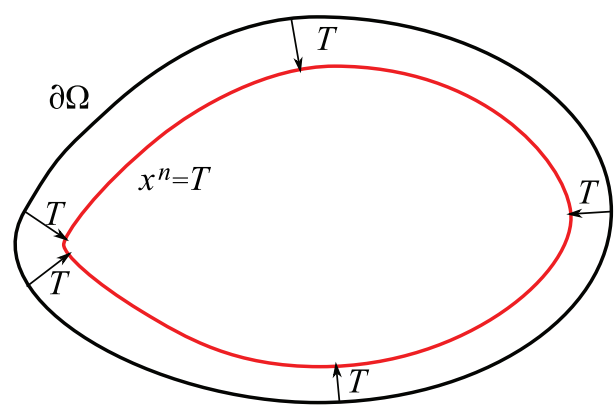

Figure 1. Theorem 2.2 
Theorem 2.2. Assume that $\partial \Omega$ is strictly convex. Let $T>0$ be such that $x^{n}:=$ $\operatorname{dist}(x, \partial \Omega)$ is a smooth function in $\Omega$ with non-zero differential for $0 \leq x^{n} \leq T$ and $\left\{x^{n}=s\right\}, 0 \leq s<T$ are strictly convex surfaces. Let $u$ solve (11) and the function a satisfies (13) for $0 \leq x^{n} \leq T$. Also assume the regularity conditions (10). Then if $u$ has zero Cauchy data on $(-T, T) \times \partial \Omega$, we also have

$$
F(x)=0 \quad \text { for } x \in \Omega, \operatorname{dist}(x, \partial \Omega)<T .
$$

Proof. Let $\left(x^{\prime}, x^{n}\right)$ be normal boundary coordinates near $\partial \Omega$ with $s_{0}$ fixed. Here $x^{n}$ is the signed distance to $\partial \Omega$ so that $x^{n}>0$ in $\Omega$. The function $x^{n}$ is defined in a small neighborhood of $\partial \Omega$ while $x^{\prime}$ are local coordinates near some boundary point. The metric $g$ then takes the form

$$
g_{\alpha \beta}\left(x^{\prime}, x^{n}\right) \mathrm{d} x^{\alpha} \mathrm{d} x^{\beta}+\left(\mathrm{d} x^{n}\right)^{2},
$$

$\alpha, \beta \leq n-1$. Given $f(x)$, independent of the dual variable $\xi$, we have

$$
G^{2} f=\left(\xi^{i} \frac{\partial}{\partial x^{i}}-\Gamma_{j k}^{i} \xi^{j} \xi^{k} \frac{\partial}{\partial \xi^{i}}\right) \xi^{\ell} \frac{\partial}{\partial x^{\ell}} f=\frac{\partial^{2} f}{\partial x^{i} \partial x^{j}} \xi^{i} \xi^{j}-\Gamma_{j k}^{i} \xi^{j} \xi^{k} \frac{\partial f}{\partial x^{i}} .
$$

Now assume that $f$ is a function of $x^{n}$ only. Then in the term involving $\Gamma_{j k}^{i}$, only $\Gamma_{j k}^{n}$ will remain. On the other hand, $\Gamma_{j k}^{n}=-\frac{1}{2} \partial g_{j k} / \partial x^{n}$ is the second fundamental form II $>0$ of the level sets of $x^{n}$, w.r.t. the chosen orientation, and it is zero when either $j=n$ or $k=n$; see, e.g., [20, p. 113].

Restrict (19) to $\partial \Omega=\left\{x^{n}=0\right\}$ to get

$$
\left.G^{2} f\right|_{x^{n}=0}=\left.\left(f^{\prime \prime}\left(x^{n}\right)\left(\xi^{n}\right)^{2}-\operatorname{II}\left(x^{\prime}\right)\left(\xi^{\prime}, \xi^{\prime}\right) f^{\prime}\left(x^{n}\right)\right)\right|_{x^{n}=0} .
$$

To satisfy (8) for $f=f\left(x^{n}\right)$, we need

$$
f^{\prime \prime}(0) \geq 1, \quad-f^{\prime}(0) \geq R,
$$

where $1 / R$ is the minimum over $\partial \Omega$ of the smallest eigenvalue (principal curvature) of the second fundamental form II. We can think of $R$ as the largest curvature radius of $\partial \Omega$ and, by assumption, $0<R<\infty$. Then $f=r^{2} / 2$ with the following function satisfies (8):

$$
r(x)=R-x^{n},
$$

because then $f:=r^{2} / 2=\left(R-x^{n}\right)^{2} / 2$ clearly satisfies (20). Therefore, the function

$$
\psi_{p}(t, x):=\left(R-x^{n}\right)^{2}-\delta t^{2}-p
$$

generates a strongly pseudo-convex $\phi_{p}$, with the assumption that $\left\{\psi_{p}=0\right\}$ is noncharacteristic. Also, the last inequality in (12) holds. Note that in Example 1, if $\partial \Omega=\left\{x ;|x|=R_{0}\right\}$, then $x^{n}=R_{0}-|x|$, and one can choose $R=R_{0}$. Then $\psi_{p}=|x|^{2}-\delta t^{2}-p$, which is the phase function in the example. As in the example, we can show that $\left\{\psi_{p}=0\right\}$ is non-characteristic for $p>0$. In fact, we will work locally near $x^{n}=t=0$, and $p=R$, and clearly, $\psi_{p}$ is non-characteristic there.

Fix $0<\varepsilon \ll 1$, and let $0<x^{n}<\varepsilon$. We restrict $p$ to the interval $(R-\varepsilon)^{2} \leq p \leq$ $R^{2}$. This choice of the phase function corresponds to pseudo-convex surfaces given by

$$
\left(R-x^{n}\right)^{2}-\delta t^{2}=p, \quad(R-\varepsilon)^{2} \leq p \leq R^{2}, \quad 0 \leq x^{n} \leq \varepsilon<R .
$$

In $\mathbf{R}_{t} \times \bar{\Omega}_{x}$, this restricts $t$ to $|t|=O(\sqrt{\varepsilon})$; see also (23) below. We will apply Theorem 2.1 with the phase function $\phi_{p}:=\exp \left(\mu \psi_{p}\right)-1, \mu \gg 1$, with $p$ as in (22). 
On $\mathcal{G}:=(-T, T) \times \partial \Omega$, we have $\left.\psi_{p}\right|_{x^{n}=0}=R^{2}-\delta t^{2}-p$. To have $\phi_{p}<0$ in $\partial Q \backslash \mathcal{G}$, we need $\psi_{p}<0$ there, and therefore $R^{2}-\delta T^{2}<p$ for all $p$ as in (22). Therefore, if

$$
T>\sqrt{R^{2}-(R-\varepsilon)^{2}}=\sqrt{2 \varepsilon R}+O\left(\varepsilon^{2}\right),
$$

which is always true when $\varepsilon \ll 1$, we can apply the theorem for $\delta<1$ close enough to 1 . Therefore, we get that if $u$ has zero Cauchy data on $(-T, T) \times \partial \Omega$ with $T$ as in (23), then $F(x)=0$ for $\psi_{p}(0, x)>0$ for any $s$ as before, i.e., for $0 \leq x^{n}<\varepsilon$. Note that this does not prove the theorem yet, even when $T$ is small enough so that we can have equality in (23) with some $\varepsilon$ satisfying the smallness requirements. The reason is that we get $F=0$ in a much smaller region: $0 \leq x^{n} \leq \varepsilon$ instead of $0 \leq x^{n} \leq T$ because for small $\varepsilon$, we have $T \sim \sqrt{\varepsilon} \gg \varepsilon$. Also, $T \gg 1$ when $R$ is large, i.e., when $\partial \Omega$ is close to a flat surface at some point and direction. In other words, the price that we pay with $T$ to push $\operatorname{supp} F$ by $\varepsilon$ depends on the (largest) radius of the curvature of $\partial \Omega$, and this is not what we are trying to prove. We will use this argument as an incremental step only, and will prove the theorem by applying a finite number of such steps.

To get the sharp time $T$, not necessarily small, we notice that we just proved that if $u$ has zero Cauchy data on $(-T, T) \times \partial \Omega$ with $T$ as in (23) and $\varepsilon \ll 1$, then $F(x)=0$ for $x \in \Omega, \operatorname{dist}(x, \partial \Omega)<\varepsilon$. Then $P u=0$ in the same domain and $|t|<T$, by (11). By unique continuation, see Proposition 2.1

$$
u(t, x)=0 \quad \text { for } x \in \Omega, \operatorname{dist}(x, \partial \Omega)+|t|<T, \operatorname{dist}(x, \partial \Omega) \leq \varepsilon .
$$

In particular,

$$
\partial_{x}^{\alpha} u(t, x)=0 \quad \text { for } x \in \Omega, \operatorname{dist}(x, \partial \Omega)=\varepsilon,|t|<T-\varepsilon,|\alpha| \leq 1,
$$

provided that $T>\varepsilon$, thus we have zero Cauchy data there.

Let $\tilde{\varepsilon}$ be the supremum of all $\varepsilon$ for which the following statement holds: if $u$ has zero Cauchy data on $(-T, T) \times \partial \Omega$, then $F(x)=0$ when $\operatorname{dist}(x, \partial \Omega)<\varepsilon$. Then $\tilde{\varepsilon}$ has that property as well. If $\tilde{\varepsilon}<T$, then by the argument above, see (25), $u$ has zero Cauchy data on $(-T+\tilde{\varepsilon}, T-\tilde{\varepsilon}) \times \partial \Omega$. Then we can repeat the argument leading to (24) to reduce supp $f$ even further, by applying Theorem 2.1 to the domain $\{x \in \Omega$, $\operatorname{dist}(x, \partial \Omega) \geq \varepsilon\}$ which has a strict convex boundary by assumption. This would contradict the choice of $\tilde{\varepsilon}$. Therefore, $\tilde{\varepsilon}=T$.

Recall that given two subsets $A$ and $B$ of a metric space, the distance $\operatorname{dist}(A, B)$ is defined by

$$
\operatorname{dist}(A, B)=\sup (\operatorname{dist}(a, B) ; a \in A) .
$$

This function is not symmetric in general, and its symmetrizer is called a Hausdorff distance, defined as

$$
\operatorname{dist}_{\mathrm{H}}(A, B)=\max (\operatorname{dist}(A, B), \operatorname{dist}(B, A)) .
$$

Let $\Omega_{1} \ni \Omega$ be another domain with smooth boundary so that $\partial \Omega_{1}$ is given by $\operatorname{dist}(x, \partial \Omega)=\varepsilon \ll 1$. Let $\Sigma_{s}, s_{1} \leq s \leq s_{2}$ be a continuous family of compact oriented surfaces in $\Omega_{1}$. By a continuous family, we mean a family such that the Hausdorff distance $\operatorname{dist}_{\mathrm{H}}\left(\Sigma_{s}, \Sigma_{s_{0}}\right)$ tends to 0 , as $s \rightarrow s_{0}, \forall s_{0}$. Examples also include surfaces that are not closed in $\bar{\Omega}$ but can be extended as closed ones in the larger domain $\Omega_{1}$ with the extension being in $\Omega_{1} \backslash \bar{\Omega}$. We assume that each $\Sigma_{s}$ divides $\Omega_{1}$ into two (open) connected parts: one, in the direction of the normal giving the orientation on $\Sigma_{s}$, that we denote by $\Sigma_{s}^{\text {int }}$ and the other one that contains $\partial \Omega_{1}$, 
that we denote by $\Sigma_{s}^{\text {ext }}$. We refer to Figure 2 for an illustration. We also assume that the orientation depends continuously on $s$, in the sense that for all $s_{0}$ and $x$, if $x \in \Sigma_{s}^{\text {int }}$ for $s=s_{0}$, then this is also true for $s$ close enough to $s_{0}$.

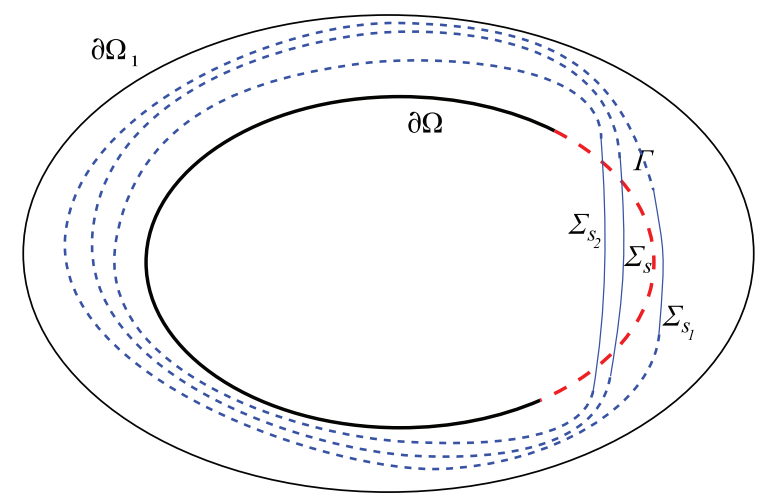

Figure 2. A typical example of the family $\Sigma_{s}$. The surfaces $\Sigma_{s}$ do not need to be closed, and the dashed parts show how they can be extended to closed surfaces. For each $\Sigma_{s}, \Sigma_{s}^{\text {int }}$ is the interior, while $\Sigma_{s}^{\text {ext }}$ is the exterior of the surface.

Let $\Gamma \subset \partial \Omega$ be a relatively open subset of $\partial \Omega$. Set

$$
\mathcal{G}:=\{(t, x) ; x \in \Gamma,|t|<\tau(x)\},
$$

where $\tau$ is a fixed continuous function on $\Gamma$. This corresponds to measurements taken at each $x \in \Gamma$ for the time interval $|t|<\tau(x)$. One special case is $\tau(x) \equiv T$, for some $T>0$; then $\mathcal{G}=(-T, T) \times \Gamma$.
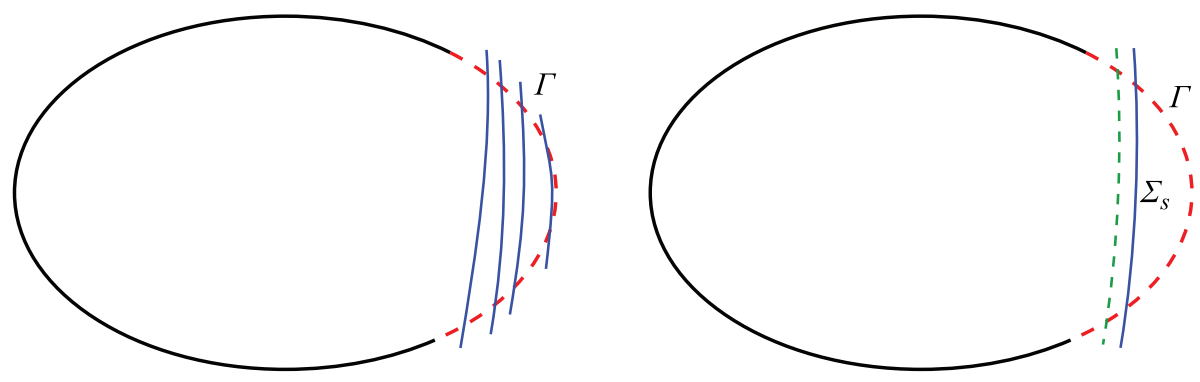

Figure 3. An illustration to Theorem 2.3 and its proof. Left: the family $\Sigma_{s}$. Right: the "inductive" step of the proof. The curve on the right is $\Sigma_{s}$; the dashed one on the left is at distance $\varepsilon$ from $\Sigma_{s}$.

Theorem 2.3. Let $\Omega_{1}, \mathcal{G}$, and $\Sigma_{s}, s_{1} \leq s \leq s_{2}$, be as above. Let $u$ solve (11) and have zero Cauchy data on $\mathcal{G}$. Let the regularity conditions (10) and the ellipticity condition (13) be satisfied on $K:=\left(\bigcup_{s_{1} \leq s \leq s_{2}} \Sigma_{s}\right) \cap \bar{\Omega}$. Assume that

(a) $\operatorname{supp} F \subset \Sigma_{s_{1}}^{\text {int }}$

(b) $\Sigma_{s} \cap \bar{\Omega}$ is strictly convex for any $s$,

(c) $\Sigma_{s} \cap(\partial \Omega \backslash \Gamma)=\emptyset$ for any $s$. 
Also assume that

$$
\text { for any } x \in \Sigma_{s} \cap \bar{\Omega} \text {, there is } y \in \Gamma \text { so that } \tau(y)>\operatorname{dist}(x, y) \text {. }
$$

Then

$$
\operatorname{supp} F \cap \Sigma_{s}=\emptyset, \quad \forall s .
$$

Proof. Extend $F$ as zero outside $\Omega$. Fix $s \in\left[s_{1}, s_{2}\right]$, and assume that

$$
\operatorname{supp} F \subset \overline{\sum_{s}^{\text {int }}} \text {. }
$$

By (28), for any $x \in \Sigma_{s} \cap \bar{\Omega}, \tau(y)>\operatorname{dist}(x, y)$ for some $y \in \Gamma$. By the unique continuation statement of Proposition 2.1 $u$ vanishes near that $x$ for small $|t|$; and, in particular, $u$ has zero Cauchy data on $(-\delta, \delta) \times \Sigma_{s} \cap \bar{\Omega}$ for $0 \leq \delta \ll 1$. By assumption, this is also true on $(-\delta, \delta) \times \Gamma$.

Let $x^{n}$ be a boundary normal coordinate to $\Sigma_{s}$ positive in $\Sigma_{s}^{\text {int }}$. Let $\psi(t, x)$ be as in (21), and $\varepsilon>0$ be as in the proof of Theorem 2.2. The function $\phi=\exp (\mu \phi)$, $\mu \gg 1$, is guaranteed to be pseudo-convex only for $x$ in an $O(\varepsilon)$ neighborhood of $\Sigma_{s} \cap \bar{\Omega}$, and for $|t| \leq \delta=O(\sqrt{\varepsilon})$, if $\varepsilon \ll 1$, by (b). We apply Lemma 2.1 to the set $D=(-\delta, \delta) \times \Sigma_{s}^{\text {int }} \cap \Omega$ to conclude by (b) and (c), as in the proof of Theorem 2.1. that $F=0$ in some neighborhood of $\Sigma_{s} \cap \bar{\Omega}$; see Figure 3 .

Now let $s_{0}$ be the supremum of all $s \in\left[s_{1}, s_{2}\right]$ for which (30) holds. The latter set is non-empty, by (a). By the continuity of $s \mapsto \Sigma_{s}$, (30) holds for $s=s_{0}$. Indeed, assuming that (30) does not hold for $s=s_{0}$, we can find $0<\varepsilon \ll 1$, so that (30) does not hold for $s_{0}-\varepsilon \leq s \leq s_{0}$. That contradicts the choice of $s_{0}$ to be the least upper bound. On the other hand, by what we proved above, $s_{0}$ cannot be an upper bound, unless $s=s_{2}$. This completes the proof.

Remark 2.1. The purpose of condition (28) is to guarantee that any point $x$ where we want to prove $F(x)=0$ is reachable from $\Gamma$ (from some point $y$ ) at a time not exceeding $\tau(y)$. In other words, there is a "signal" (a unit speed curve) issued from $x$ that will reach the observation part $\Gamma$ of $\partial \Omega$ at a time while we are still making measurements there. By finite speed of propagation, it is a necessary condition as well.

Remark 2.2. A sufficient but an easier to formulate condition to replace (28) is

$$
\mathcal{G}=(-T, T) \times \Gamma \quad \text { with } \quad T>\max _{s} \operatorname{dist}\left(\Sigma_{s} \cap \bar{\Omega}, \Gamma\right) ;
$$

see (26). An even simpler sufficient condition is

$$
\mathcal{G}=(-T, T) \times \Gamma \quad \text { with } \quad T>\operatorname{dist}(\Omega, \Gamma)
$$

\subsection{Examples.}

Example 2. Let $\Omega \subset \mathbf{R}^{2}$ be a bounded domain, and let $g$ be a metric on $\bar{\Omega}$. Assume that $\partial \Omega$ is convex in the metric, and that there is $x_{0} \in \partial \Omega$, so that all geodesics issued from $\partial \Omega$, pointing into $\bar{\Omega}$, exit $\bar{\Omega}$ after some fixed time. That property does not depend on the way we extend $g$ outside $\bar{\Omega}$. All simple (see 22 for a definition) $(\Omega, g)$ have this property, and all non-trapping convex ones have it too. We will show that in this case we can cover $\bar{\Omega}$ by a foliation of smooth surfaces (curves, actually) $\Sigma_{s}$ that are a small perturbation of the geodesics through $x_{0}$.

Extend $g$ in a small neighborhood $\Omega_{1}$ of $\bar{\Omega}$, and let $y_{0} \notin \bar{\Omega}$ be close to $x_{0}$ so that the geodesics through $y_{0}$ have the same property but in $\Omega_{1}$. Choose global coordinates in the latter as normal coordinates centered at $y_{0}$. In those coordinates, 


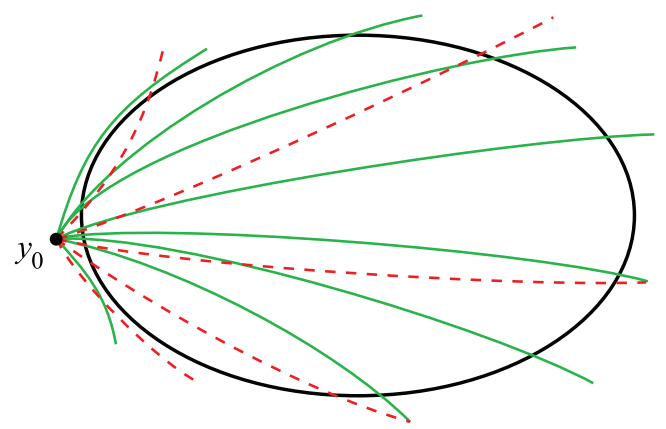

Figure 4. Example 2

the geodesics through $y_{0}$ are the lines, i.e., they solve $x^{\prime \prime}=0$. At each $x$, let $J$ be the rotation operator in the tangent space given by $J v=\left(-v_{2}, v_{1}\right)$, where we used the standard index raising/lowering convention, and the vector $J v$ is identified with the covector on the right-hand side. Given $0<\delta \ll 1$, define the curves $\Sigma_{s}$ as solutions of $x^{\prime \prime}=-\delta J x^{\prime}$. The parameter $s$ measures the angle of the initial direction at $y_{0}$ with a fixed direction. Then $\Sigma_{s}$ are strictly convex when viewed from the side determined by the normal $J x^{\prime}$. In Figure 4, this is the upper side. We can always extend the curves $\Sigma_{s}$ to closed ones with the extension being outside $\Omega$. Assume now that $u$ has zero Cauchy data. Then we can apply Theorem 2.3 to conclude that $F=0$ when $T$ is appropriately chosen. A possible choice of $T$ is the diameter of $\bar{\Omega}$, given by $\max (\operatorname{dist}(x, y) ; x, y \in \bar{\Omega})$. To optimize $T$, we can consider a similar family, with $\delta$ negative. In Figure 4 , they are shown as dashed curves. Then the "interior" and the "exterior" are reversed. The two families converge to the set of the geodesics through $y_{0}$, as $|\delta| \rightarrow 0$. Then the value for $T$ is enough to apply the theorem and can be obtained by finding a geodesic $\gamma_{0}$ through $y_{0}$ so that maximum of the distances from $\gamma_{0} \cap \bar{\Omega}$ to the upper and the lower side of $\partial \Omega$ is maximized; then $T$ is that value.

Let $\Omega$ be an ellipse, and let $g$ be Euclidean. If $y_{0}$ is one of the vertices on the major (minor) axis, then $\gamma_{0}$ is the major (minor) axis, and it is enough to take $T$ to be a half of the length of the major (minor) axis.

Example 3. Let $\Omega \subset \mathbf{R}^{2}$ be as above. Assume that there exists a closed non-selfintersecting geodesic $\gamma_{0}$ of the metric $g$. Assume that the region between $\partial \Omega$ and $\gamma_{0}$ can be foliated by a continuous family of strictly convex curves $\Sigma_{s}$. Then $\operatorname{supp} F$ is contained inside $\gamma_{0}$, if $T=\operatorname{dist}\left(\gamma_{0}^{\text {ext }}, \partial \Omega\right)$, where $\gamma_{0}^{\text {ext }}$ is the exterior of $\gamma_{0}$ in $\Omega$. Our analysis does not allow us to extend the equality $F=0$ inside.

Example 4. Let $\Omega \subset \mathbf{R}^{2}$ be diffeomorphic to a disk, and let $\Gamma$ be a relatively open connected part of $\partial \Omega$. Fix a metric $g$ in some neighborhood of $\bar{\Omega}$. We do not need to assume that the whole $\partial \Omega$ is convex but we will assume that there is a continuous family of geodesics, with endpoints outside $\bar{\Omega}$, covering the region between $\Gamma$ and the geodesic connecting the endpoints of $\Gamma$; see also Figure 2, In Figure 6, this is the unshaded region. The latter assumption is fulfilled if for example one of those points has the property that all geodesics issued from it, and pointed inside $\Omega$, leave the unshaded region after some fixed time. In particular, $(\Omega, g)$ being nontrapping suffices. Then we can perturb those geodesics to curves that are convex 


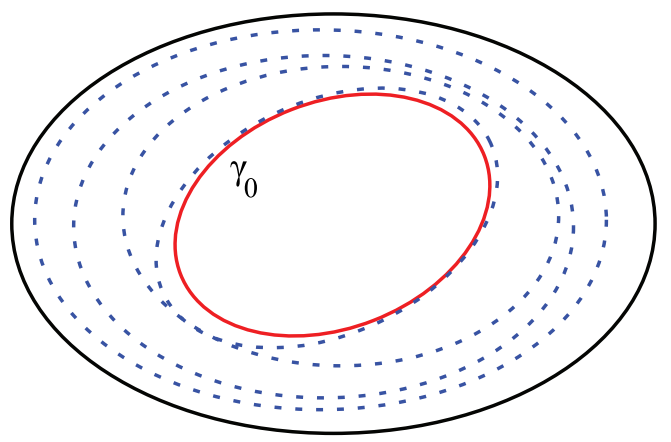

Figure 5. Example 3

(bending to the shaded region), as in Example 2, to show that when $T$ is chosen in an appropriate way, $\operatorname{supp} F$ must be in the shaded region.

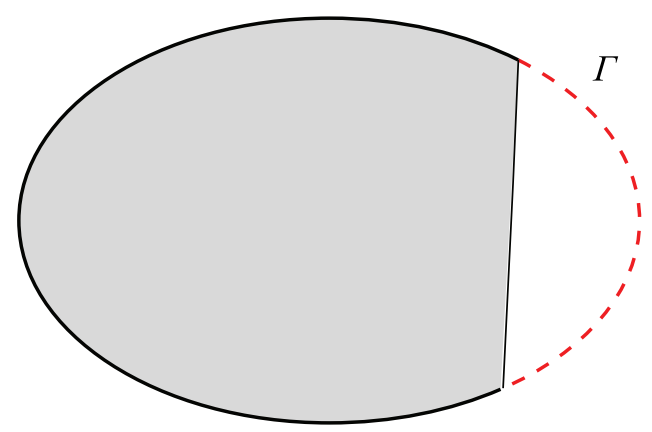

Figure 6. Example 4

A higher dimensional analog of this example would be $\Omega$ diffeomorphic to a ball with $\Gamma \subset \partial \Omega$ diffeomorphic to a disk on $\partial \Omega$. Then $F=0$ in the region covered by families of convex surfaces. For example, let $\Omega$ be the ellipsoid $\sum\left(x^{i}\right)^{2} / a_{i}^{2}=1$, and let $g$ be Euclidean. Let $\Gamma=\partial \Omega \cap\left\{x^{1}>C\right\}$ with $0<C<a_{1}$. Then $F=0$ in $\Omega \cap\left\{x^{1}>C\right\}$, and it is enough to choose $T=a_{1}-C ; T$ may or may not be sharp, depending on all $a_{j}$ and $C$.

\section{A NON-LINEAR PROBLEM OF RECOVERY OF A SPEED} With one measurement. Applications to thermoacoustics

In section 2, we showed that one can uniquely recover $f$ when $t$ varies over a symmetric interval $[-T, T]$, and $\left.u\right|_{t=0}$ is known. No knowledge of $\left.u_{t}\right|_{t=0}$ is required. Now assume that $t$ varies over the interval $[0, T]$, and $a, u$ admit even extensions for $t \in[-T, T]$ of regularity as in the preceding section. In particular, this means that $\left.u_{t}\right|_{t=0}=0$. In other words, $u$ solves

$$
\left\{\begin{aligned}
P u & =a(t, x) F(x) & & \text { in }(0, T) \times \Omega, \\
\left.u\right|_{t=0} & =0 & & \text { in } \Omega, \\
\left.u_{t}\right|_{t=0} & =0 & & \text { in } \Omega,
\end{aligned}\right.
$$


compare with (2). Then one has obvious corollaries of the results of the previous section that we are not going to formulate. One can interpret those results as a recovery of a source, given $a$. If $a=1$, then one can differentiate the equation above w.r.t. $t$ and to reduce the problem to recovery of an initial condition for the homogeneous wave equation, that is, the classical thermoacoustic problem of recovering $f$ given $\Lambda f$, with a known speed; see (34) below.

3.1. The thermoacoustic model. Let $u$ solve problem (1), where $c=c(x)>0$ is smooth and $T>0$ is fixed. Note that the wave equation is now solved in the whole $\mathbf{R}^{n}$.

Assume that $f$ is supported in $\bar{\Omega}$, where $\Omega \subset \mathbf{R}^{n}$ is some smooth bounded domain. Also assume that $c=1$ outside $\Omega$. This is not an essential assumption; it is enough for $c$ to be known and fixed outside $\Omega$. The measurements are modeled by the operator

$$
\Lambda f:=\left.u\right|_{[0, T] \times \partial \Omega} .
$$

The problem is to reconstruct the unknown $c$ and $f$, if possible. We will now study the case when $f$ is known, and we want to reconstruct $c$.

This inverse problem models thermoacoustic and photoacoustic tomography, where a microwave or a laser impulse is sent to a patient's body and we measure the generated acoustic wave outside the body. We refer to [3, 4, 15, 6, 13, 16, 23, 24, and the references there, for some mathematical works in this direction.

3.2. Uniqueness results for the linear problem. Let $(c, f),(\tilde{c}, \tilde{f})$ be two pairs, and let $u, \tilde{u}$ be the corresponding solutions of (1). Then

$$
\left\{\begin{aligned}
\left(\partial_{t}^{2}-c^{2} \Delta\right)(\tilde{u}-u) & =\left(\tilde{c}^{2}-c^{2}\right) \Delta \tilde{u} \quad \text { in }(0, T) \times \mathbf{R}^{n}, \\
\left.(\tilde{u}-u)\right|_{t=0} & =0, \\
\left.\partial_{t}(\tilde{u}-u)\right|_{t=0} & =0 .
\end{aligned}\right.
$$

Then

We have

$$
(\tilde{\Lambda}-\Lambda) f=\left.(\tilde{u}-u)\right|_{[0, T] \times \partial \Omega}
$$

$$
(\delta \Lambda) f:=(\tilde{\Lambda}-\Lambda) f=\left.w\right|_{[0, T] \times \partial \Omega},
$$

where $w$ solves (2) with $F$ and $a$ as in (3). Then supp $F \subset \bar{\Omega}$, and given the regularity of $c$ and $\tilde{c}$, we also have $F=0$ on $\partial \Omega$. The measurement (36) however determines the Dirichlet data on $[0, T] \times \partial \Omega$ only. The Neumann data can be recovered from that however; see also [24, sec. 7] and Lemma 3.1 below. We emphasize again that $w$ solves the wave equation for $x$ in the whole $\mathbf{R}^{n}$, and this is what allows us to recover the Neumann data.

We assume below that $w$ solves the more general problem

$$
\left\{\begin{aligned}
\left(\partial_{t}^{2}-\Delta_{g}\right) w & =a(t, x) F(x) \quad \text { in }(0, T) \times \mathbf{R}^{n}, \\
\left.w\right|_{t=0} & =0 \\
\left.\partial_{t} w\right|_{t=0} & =0
\end{aligned}\right.
$$

where $g$ is a smooth Riemannian metric that is Euclidean outside $\Omega$. In some of the results below, we do not assume that $F$ and $a$ are given by (3).

We introduce the energy space associated with the wave equation below, to be able to deal both with metrics and variables speeds. Write the metric $g$ as $g=c^{-2} g_{0}$, where $c(x)>0$. In the thermoacoustic case, one usually takes $g_{0}$ to be 
Euclidean. Let $\Delta_{g_{0}}$ be the Laplace-Beltrami operator as above but related to $g_{0}$. Modulo lower order terms, $c^{2} \Delta_{g_{0}}$ and $\Delta_{g}$ coincide. Write $P$ in the form

$$
P=\partial_{t}^{2}-A, \quad A=c^{2} \Delta_{g_{0}}+\text { lower order terms, }
$$

compare with (4). Notice first that $c^{2} \Delta_{g_{0}}$ is formally self-adjoint w.r.t. the measure $c^{-2} \mathrm{~d}$ Vol. Given a domain $U$, and a function $u(t, x)$, define the energy

$$
E_{U}(t, u)=\int_{U}\left(\left|\nabla_{x} u\right|_{0}^{2}+c^{-2}\left|u_{t}\right|^{2}\right) \mathrm{d} \operatorname{Vol}_{0}(x),
$$

where $\left|\nabla_{x} u\right|_{0}$ is the norm in the metric $g_{0}$, and $\mathrm{d} \mathrm{Vol}_{0}$ is the volume measure w.r.t. $g_{0}$ as well. In particular, we define the space $H_{D}(U)$ to be the completion of $C_{0}^{\infty}(U)$ under the Dirichlet norm

$$
\|f\|_{H_{D}}^{2}=\int_{U}\left|\nabla_{x} u\right|_{0}^{2} \mathrm{~d} \operatorname{Vol}_{0}(x) .
$$

It is easy to see that $H_{D}(U) \subset H^{1}(U)$, if $U$ is bounded with smooth boundary; therefore, $H_{D}(U)$ is topologically equivalent to $H_{0}^{1}(U)$. If $U=\mathbf{R}^{n}$, this is true for $n \geq 3$ only. By the finite speed of propagation, the solution with compactly supported Cauchy data always stays in $H^{1}$ even when $n=2$. The energy norm for the Cauchy data $\left[f_{1}, f_{2}\right]$, that we denote by $\|\cdot\|_{\mathcal{H}}$ is then defined by

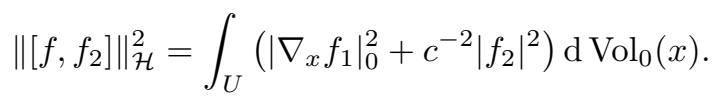

This defines the energy space

$$
\mathcal{H}(U)=H_{D}(U) \oplus L^{2}(U) .
$$

Here and below, $L^{2}(U)=L^{2}\left(U ; c^{-2} \mathrm{~d} \mathrm{Vol}_{0}\right)$. Note also that

$$
\|f\|_{H_{D}}^{2}=(-A f, f)_{L^{2}} .
$$

The wave equation then can be written as the system

$$
\mathbf{u}_{t}=\mathbf{A u}, \quad \mathbf{A}=\left(\begin{array}{ll}
0 & I \\
A & 0
\end{array}\right),
$$

where $\mathbf{u}=\left[u, u_{t}\right]$ belongs to the energy space $\mathcal{H}$. The operator $\mathbf{A}$ then extends naturally to a skew-selfadjoint operator on $\mathcal{H}$. We denote by $\mathbf{U}(t)$ the group $\exp (t \mathbf{A})$. In this paper, we will deal with either $U=\mathbf{R}^{n}$ or $U=\Omega$. In the latter case, the definition of $H_{D}(U)$ reflects Dirichlet boundary conditions.

Next we will define the outgoing DN map. Given $g \in C_{0}^{\infty}((0, \infty) \times \partial \Omega)$, let $w$ solve the exterior mixed problem with $c=1$ :

$$
\left\{\begin{aligned}
\left(\partial_{t}^{2}-\Delta\right) v & =0 \\
\left.v\right|_{[0, T] \times \partial \Omega} & =g \\
\left.v\right|_{t=0} & =0 \\
\left.\partial_{t} v\right|_{t=0} & =0
\end{aligned} \text { in }(0, T) \times \mathbf{R}^{n} \backslash \bar{\Omega}\right.
$$

Then we set

$$
N g=\left.\frac{\partial w}{\partial \nu}\right|_{[0, T] \times \partial \Omega} .
$$

By [14], for $g \in H_{(0)}^{1}([0, T] \times \partial \Omega)$, we have $\left[w, w_{t}\right] \in C([0, T) ; \mathcal{H}(\Omega))$; therefore,

$$
N: H_{(0)}^{1}([0, T] \times \partial \Omega) \rightarrow C\left([0, T] \times H^{\frac{1}{2}}(\partial \Omega)\right)
$$


is continuous, where the subscript (0) indicates the closed subspace of functions vanishing at $t=0$. Note that the results in [14] require the domain to be bounded but, by finite domain of dependence, we can remove that restriction in our case. We also refer to [3, Proposition 2] for a sharp domain of dependence result for exterior problems.

When $F$ and $a$ are given by (3), the next lemma follows directly from its version [24, sec. 7] for $\Lambda f$ by subtracting $\tilde{\Lambda} f$ and $\Lambda f$.

Lemma 3.1. Let $w$ solve (2) with $F$ supported in $\bar{\Omega}$, and let $t \mapsto a(t, \cdot) F(\cdot) \in L^{2}(\Omega)$ be continuous. Assume that $c=1$ outside $\Omega$. Then for any $T>0,\left.w\right|_{[0, T] \times \partial \Omega}$ determines uniquely the normal derivative of $w$ on $[0, T] \times \partial \Omega$ as follows:

$$
\left.\frac{\partial w}{\partial \nu}\right|_{[0, T] \times \partial \Omega}=N\left(\left.w\right|_{[0, T] \times \partial \Omega}\right) .
$$

Proof. Let $v$ be the solution of (42) with $g=\left.w\right|_{[0, T] \times \partial \Omega}$. The latter is in $H_{(0)}^{1}([0, T] \times \partial \Omega)$. Let $w$ be the solution of (2). Then $v-w$ solves the unit speed wave equation in $[0, T] \times \mathbf{R}^{n} \backslash \Omega$ with zero Dirichlet data and zero initial data. Therefore, $v=w$ in $[0, T] \times \mathbf{R}^{n} \backslash \Omega$.

With this in mind, we have the following version of Theorem 2.2 in this context. Notice that in the two theorems below, we do not assume $F$ and $a$ to be given by (3).

Theorem 3.1. Assume that $\partial \Omega$ is strictly convex, $w$ solves (2) and the function a satisfies the elliptic condition (13) in the closure of the set (44) below. Let a and $w$ admit even extensions satisfying (10). Let $T>0$ be such that $x^{n}:=\operatorname{dist}(x, \partial \Omega)$ is a well-defined smooth function in $\Omega$ with non-zero differential; $\left\{x^{n}=s\right\}, 0 \leq s<T$ are strictly convex surfaces. Then if $w=0$ on $[0, T] \times \partial \Omega$, we also have

$$
F(x)=0 \quad \text { for } x \in \Omega, \operatorname{dist}(x, \partial \Omega)<T .
$$

Proof. We first apply Lemma 3.1 to conclude that the normal derivative of $w$ vanishes on $[0, T] \times \partial \Omega$ as well. The assumptions of the theorem imply that $a(t, x)$ can be extended in an even way satisfying the assumptions of Theorem 2.2 . Then $w$ admits an even extension, as a solution of the wave equation, and thus we apply Theorem 2.2

Since recovery of $F$ in (2) from $\left.w\right|_{[0, T] \times \partial \Omega}$ is a linear problem, we also get uniqueness for that problem in the set (44). We also get unique recovery of the speed $c$ in the region in (44). Those are in fact partial cases of Theorems 3.2 and 3.3 .

The local data result, in the spirit of Theorem 2.3. is not so straightforward because the recovery of the Neumann data is not so direct.

For any $(\hat{t}, \hat{x}) \in \mathbf{R}_{+} \times \partial \Omega$, define the "cone"

$$
C_{\hat{t}, \hat{x}}:=\left\{(t, x) \in \mathbf{R}_{+} \times \partial \Omega ; t+\operatorname{dist}_{0}(x, \hat{x}) \leq \hat{t}\right\},
$$

where for $a, b$ in $\mathbf{R}^{n} \backslash \Omega$, $\operatorname{dist}_{0}(a, b)$ is the infimum of the lengths of all smooth curves lying in $\mathbf{R}^{n} \backslash \Omega$ that connect $a$ and $b$.

Theorem 3.2. Let $\Omega, \Omega_{1}, \mathcal{G}$ and $\Sigma_{s}$ be as in Theorem 2.3. Let $w$ solve (2) and let $a, w$ have even extensions which satisfy (10) and (13) with $K$ being the closure 
of the set in (46) below. Assume that $w=0$ on $\mathcal{G}_{+}:=\mathcal{G} \cap\{t \geq 0\}$. Then

$$
\operatorname{supp} F \cap\left\{x \in \Omega \cap\left(\bigcup_{s} \Sigma_{s}\right) ; \exists y \in \Gamma, C_{\operatorname{dist}(x, y), y} \subset \mathcal{G}_{+}\right\}=\emptyset .
$$

Proof. Outside $\mathbf{R} \times \Omega, w$, extended in an even way for $t<0$, solves the wave equation $P w=0$ with zero Cauchy data for $t=0$ and zero Dirichlet data on $\mathcal{G}$; see (27). This does not allow us immediately to conclude that the Neumann data vanishes there too. On the other hand, by the finite domain of dependence result in $\left[3, \partial w / \partial \nu=0\right.$ near $(\hat{t}, \hat{x}) \in \mathcal{G}_{+}$, if the "cone" $C_{\hat{t}, \hat{x}}$ is contained in $\mathcal{G}_{+}$. Fix such $(\hat{t}, \hat{x})$. We apply Theorem 2.3 with $\mathcal{G}$ replaced by $C_{\hat{t}, \hat{x}}$, extended in an even way for $t<0$. This cone is a set of the type $\mathcal{G}$, see (27), with $\tau$ replaced by $\hat{\tau}(x)=\max \left(0, \hat{t}-\operatorname{dist}_{0}(x, \hat{x})\right)$, well-defined on $\Gamma$. By Theorem 2.3. supp $F$ does not contain $x \in \bar{\Omega} \cap\left(\bigcup_{s} \Sigma_{s}\right)$ so that $\hat{\tau}(y)>\operatorname{dist}(x, y)$ for some $y \in \Gamma$. The latter condition can be written as

$$
\hat{t}-\operatorname{dist}_{0}(y, \hat{x})>\operatorname{dist}(x, y) \quad \text { for some } y \in \Gamma \text {. }
$$

Now, choose $x$ as in the second set in (46), and let $y$ be the corresponding point on $\Gamma$. Set $\hat{t}=\operatorname{dist}(x, y)+\varepsilon, \hat{x}=y$. Then the cone condition $C_{\hat{t}, \hat{x}} \subset \mathcal{G}_{+}$is satisfied for $0<\varepsilon \ll 1$ because it holds for $\varepsilon=0$ by assumption, and $C_{\hat{t}, \hat{x}}$ is defined by a non-strict inequality, while $\mathcal{G}$ is defined by a strict one. Therefore, condition (47), that we just got to conclude $F=0$ near $x$, is satisfied. This completes the proof.

Remark 3.1. In case of observations on the whole boundary, i.e., when $\Gamma=\partial \Omega$, with $\mathcal{G}=[0, T] \times \partial \Omega$, (46) implies $F=0$ in the set $\operatorname{dist}(x, \partial \Omega) \leq T$. In particular,

$$
T>\operatorname{dist}(\Omega, \partial \Omega)
$$

is sufficient to conclude $F=0$; see also (32). This is in agreement with the results of the previous section since one can recover the Neumann data easily by Lemma 3.1 .

Example 5. Let $g$ be Euclidean and let $\Omega$ be strictly convex. Let $\Gamma=\left\{x^{n}>a\right\} \cap \partial \Omega$ with some fixed $a$, and let $\Omega_{a}=\left\{x^{n}>a\right\} \cap \Omega$. Then $\Omega_{a}$ satisfies the foliation condition. For any $x \in \Omega_{0}$, let $y$ be the point on $\Gamma$ with the same $x^{\prime}$ coordinates, where $x^{\prime}=\left(x^{1}, \ldots, x^{n-1}\right)$. Then $|x-y|<\operatorname{dist}_{0}(y, \partial \Omega \backslash \Gamma)$ because even when $x \in\left\{x^{n}=a\right\}$ (then $|x-y|=y^{n}-a$ is maximized), the Euclidean distance from $y$ to $\left\{x^{n}=a\right\}$ minimizes the distance from $y$ to that plane with the constraint that we take it outside $\Omega$. Then the "cone" $C_{|x-y|, y}$ is included in $\mathcal{G}_{+}$, if we choose $\mathcal{G}=(-T, T) \times \Gamma$ with $T=\operatorname{dist}(\Gamma, \partial \Omega \backslash \Gamma)$; see (26). With that choice of $\mathcal{G}$, under the assumptions of the theorem, we get $F=0$ in $\Omega_{a}$. In other words, the "cone condition" in (46) is satisfied and therefore it is not restrictive in this case.

By a perturbation argument, if $g$ is close enough to the Euclidean metric, then $F$ would vanish in a set a bit smaller than $\Omega_{0}$.

3.3. Uniqueness for the non-linear problem. We now go back to the problem of determining the sound speed $c$ in (1) from $\Lambda f$ with $f$ fixed and known. Clearly, some conditions on $f$ are needed since, when $f=0$, for example, we get no information about $c$.

Based on Theorems 3.1 or 3.2 , we can easily formulate versions for the non-linear problem. We will formulate a consequence of the latter theorem under conditions that guarantee that we can recover $c$ in the whole $\Omega$. 
Theorem 3.3. Let $c$ and $\tilde{c}$ be two smooth positive speeds equal to 1 outside $\Omega$. Let $\Sigma_{s}$ be as in Theorem 2.3 and satisfy (a) and (b) there with $F:=\tilde{c}^{2}-c^{2}$ and with the strict convexity assumption in (b) fulfilled w.r.t. the speed c. Let

$$
\tilde{\Lambda} f=\Lambda f \quad \text { on }[0, T] \times \partial \Omega, \quad \text { with } T>\max _{s} \operatorname{dist}\left(\Sigma_{s} \cap \bar{\Omega}, \partial \Omega\right) .
$$

Assume that for some compact $K \subset \bar{\Omega}$,

$$
\operatorname{supp}(\tilde{c}-c) \subset K, \quad \Delta f(x) \neq 0 \quad \text { for } x \in K .
$$

Then $\tilde{c}=c$ in $\bigcup \Sigma_{s}$.

If in particular $\bigcup \Sigma_{s}$ is dense in $K$, and $T>\operatorname{dist}(\Omega, \partial \Omega)$, then $\tilde{c}=c$.

Examples of domains $\Omega$ so that any $K \subset \bar{\Omega}$ satisfies the density condition include geodesic balls with a fixed center under the assumption that they are all strictly convex. Then $\tilde{c}=c$ everywhere except in the center, and by continuity, this is true in the center as well. In particular, this holds if $\|c\|_{C^{2}} \leq M$ with some $M>0$ fixed, and $\operatorname{diam}(\Omega) \ll 1$ because $M$ puts an upper bound of the curvature of the metric $c^{-2} \mathrm{~d} x^{2}$.

Proof. Set $w=\tilde{u}-u$, where $\tilde{u}$ and $u$ solve (11) with the speeds $\tilde{c}$ and $c$, respectively; and the same holds for $f$. Then $w$ solves (2) with $a$ and $F$ as in (3). The condition (13) is then equivalent to $\Delta \tilde{u}(0, x) \neq 0, \forall x \in K$. By (11), this is equivalent to $\Delta f(x) \neq 0, \forall x \in K$. Then an application of Theorem 3.2 completes the proof.

Remark 3.2. The condition $\Delta f(x) \neq 0$ may look mysterious at first glance. The stability analysis below shows that it is needed for the linearization to be Fredholm. A simplified look at this condition is the following. Let us remove the need for $f$ to be 0 outside $\Omega$. Then any harmonic function $f$ is also a time independent solution $u=f$ of the wave equation $\left(\partial_{t}^{2}-c^{2}(x) \Delta\right) u=0$, regardless of $c$. Then $\Lambda f$ carries no information about $c$ at all. If $f$ is harmonic only on some open geodesic ball $U$, then $u=f$ (regardless of the speed) in the light cone with base $U$, and then $a=0$ there; see (3). Then the kernel of the linearized map would be $C^{\infty}$ for $y \in U$, as it follows from (53). That implies high instability for the linearization, at least.

3.4. Stability. As a general principle, we have stability in Sobolev spaces if we can detect all singularities at $\mathcal{G}$ where we make measurements; see, e.g., [21. Under the assumptions of Theorem 3.3 , that would require that

$$
\forall(x, \xi) \in S K \text {, the geodesic through }(x, \xi) \text { hits } \partial \Omega \text { for some } t \text { with }|t|<T,
$$

where $S K$ stands for the unit sphere bundle of $(K, g)$. Here we used the fact that the problem extends in an even way w.r.t. $t$. We also identify vectors and covectors by the metric $g$.

Notice that condition (51) is stronger than the uniqueness condition (48). The latter requires that from any $x$ there is a signal (a unit speed curve) originating from $x$ reaching $\partial \Omega$ up to time $T$, i.e., $\operatorname{dist}(x, \partial \Omega)<T$. Condition (51) requires that from any $x$ and any direction $\xi$ the geodesic through it reaches $\partial \Omega$ for time $|t|<T$. The same conditions appear in the analysis of the thermoacoustic problem of recovery of $f$, given $c$ and $\Lambda f$; see [23, 18. Here however, we assume the foliation condition as well.

Let $R \mathbf{w}$ denote the trace of the first component $w$ of $\mathbf{w}:=\left[w, w_{t}\right]$, defined on $[0, T] \times \Omega$, to $[0, T] \times \partial \Omega$. When $w$ is a scalar valued function, $R w$ just denotes the trace of $w$. 
Theorem 3.4. Let $w$ solve (2) with $w$, let a satisfy the regularity assumptions of Theorem 3.1, let $F$ be supported in a compact $K \subset \Omega$, with $F \in L^{2}(K)$, and let $a(0, x) \neq 0$ for all $x \in K$. Let $\Sigma_{s}$ be as in Theorem 2.3, and assume that $\bigcup \Sigma_{s}$ is dense in $\Omega$ (the foliation condition). Let $K$ and $T$ satisfy (51) (the stability condition). Then

$$
\|F\|_{L^{2}(K)} \leq C\left\|w_{t t}\right\|_{L^{2}([0, T] \times \partial \Omega)}
$$

with a constant $C$ that remains uniform when the coefficient a stays in a fixed bounded set in $C^{2}([0, T] ; C(\bar{\Omega}))$.

Proof. We use the notation $a(t)=a(t, \cdot)$ below. Differentiate

$$
\mathbf{w}=\int_{0}^{t} \mathbf{U}(s)[0, a(t-s) F] \mathrm{d} s
$$

to get

$$
\partial_{t} R w=R \mathbf{U}(t)[0, a(0) F]+R \int_{0}^{t} \mathbf{U}(s)\left[0, a^{\prime}(t-s) F\right] \mathrm{d} s .
$$

Differentiate again and use the identity $a^{\prime}(0)=0$ and the definition of $\Lambda$ to get

$$
\begin{aligned}
\partial_{t}^{2} R w & =\Lambda a(0) F+R \int_{0}^{t} \mathbf{U}(s)\left[0, a^{\prime \prime}(t-s) F\right] \mathrm{d} s \\
& =\Lambda a(0) F+R \int_{0}^{t} \mathbf{U}(t-s)\left[0, a^{\prime \prime}(s) F\right] \mathrm{d} s .
\end{aligned}
$$

Let $\chi \in C^{\infty}([0, T])$ be such that $\chi=0$ near $T$, and $\chi=1$ on $\left[0, T_{0}\right]$, where $T_{0}<T$ is such that (48) still holds with $T$ replaced by $T_{0}$. Let $B$ be the back-projection operator defined as follows. If $v$ solves

$$
\left\{\begin{aligned}
\left(\partial_{t}^{2}-c^{2} \Delta\right) v & =0 \\
\left.v\right|_{t=T}=\left.\partial_{t} v\right|_{t=T} & =0 \\
\left.v\right|_{[0, T] \times \partial \Omega} & =h
\end{aligned}\right.
$$

then define $B h:=\left.v\right|_{t=0}$. By [23, Theorem 3], $B \chi \Lambda$ is a classical $\Psi$ DO of order 0 with principal symbol

$$
\frac{1}{2} \chi\left(\tau_{+}(x, \xi)\right)+\frac{1}{2} \chi\left(\tau_{-}(x, \xi)\right),
$$

where $\pm \tau_{ \pm} \geq 0$ are the times needed for the unit speed geodesic issued from $(x, \xi)$ to hit $\partial \Omega$. We recall that we identify vectors and covectors by the metric $g=c^{-2} \mathrm{~d} x^{2}$. Condition (51) guarantees that the symbol above is elliptic. Let $Q$, a zeroth order $\Psi D O$, be a properly supported parametrix for it, i.e., $Q B \chi \Lambda=\operatorname{Id}+K_{0}$ in a neighborhood of $K$, with $K_{0}$ smoothing. For the purpose of this proof, we only need $K_{0}: L^{2} \rightarrow H^{1}$, which can be achieved with finite smoothness requirements on $c$.

Apply $Q B \chi$ to (54) to get

$$
Q B \chi \partial_{t}^{2} R w=\left(\mathrm{Id}+K_{0}\right) a(0) F+Q B \chi \int_{0}^{t} R \mathbf{U}(t-s)\left[0, a^{\prime \prime}(s) F\right] \mathrm{d} s .
$$

For any $s \in[0, t]$, the function $\left[0, a^{\prime \prime}(s) F\right]$ belongs to the energy space $\mathcal{H}$ and is supported in $\Omega$. Then $R \mathbf{U}(t-s)$ (a more accurate notation would be $R \mathbf{U}(\cdot-s)$ ) maps that function to a function that belongs to $H_{(0)}^{1}(\mathbf{R} \times \partial \Omega)$, where the subscript (0) indicates a support disconnected from $t=0$. This is explained in [23] in the context of thermoacoustic tomography, and the reason is that $R \mathbf{U}(\cdot-s)$ is an FIO 
of order 0 with a canonical relation of graph type; see also [25]. The dependence on $s$ is continuous, therefore, the integral in (54) belongs to that space as well. By [14], $B: H_{(0)}^{1}(\mathbf{R} \times \partial \Omega) \rightarrow H_{0}^{1}(\Omega)$ is continuous. Since $Q$ is of order zero, the integral term in (55) is a compact operator of $F$ in $L^{2}(\Omega)$, mapping $L^{2}(\Omega)$ into $H^{1}(\Omega)$.

Therefore, since $\left.a(0)\right|_{K} \neq 0$,

$$
\|F\|_{L^{2}(K)} \leq C\left\|\partial_{t}^{2} R w\right\|_{L^{2}([0, T] \times \partial \Omega)}+C\left\|K_{2} F\right\|_{L^{2}(\Omega)},
$$

with $K_{2}: L^{2}(K) \rightarrow L^{2}(\Omega)$ compact. Here we have used the fact that $B$ : $L_{\text {comp }}^{2}(\mathbf{R} \times \partial \Omega) \rightarrow L^{2}(\Omega)$ also follows from [14, or from the property of $B$ to be an FIO of order 0 with a canonical relation of graph type [23. Notice now that the map $F \mapsto R w_{t t}$, from $L^{2}(K)$ to $L^{2}([0, T] \times \partial \Omega)$, is bounded by (54) and the analysis following it because the integral term there is actually a compact operator, while $F \mapsto \Lambda a(0) F$ is bounded as an FIO of order 0 with a canonical relation of graph type 25,23 . On the other hand, it is injective as well, since $w_{t t}=0$ easily implies $w=0$, and then we apply Theorem [3.3. Then by [28, Proposition A.6.7], estimate (56) implies a similar one, with a different $C$, and the last term missing.

The statement about the uniformity of $C$ does not follow directly from the last argument above because there is no control over $C$. Instead, we will perturb estimate (52). Notice first that by (54), the map $\left.C^{2}([0, T] ; C(\bar{\Omega})) \ni a \mapsto w_{t t}\right|_{[0, T] \times \partial \Omega} \in L^{2}$ is continuous. Then if $a$ and $\tilde{a}$ are two coefficients and $w, \tilde{w}$ are the corresponding solutions, we have

$$
\begin{aligned}
\|F\|_{L^{2}(K)} & \leq C\left\|w_{t t}\right\|_{L^{2}([0, T] \times \partial \Omega)} \\
& \leq C\left\|\tilde{w}_{t t}\right\|_{L^{2}([0, T] \times \partial \Omega)}+C\left\|\tilde{w}_{t t}-w_{t t}\right\|_{L^{2}([0, T] \times \partial \Omega)} \\
& \leq C\left\|\tilde{w}_{t t}\right\|_{L^{2}([0, T] \times \partial \Omega)}+C \delta\|F\|_{L^{2}(K)},
\end{aligned}
$$

where $\delta \ll 1$ when $\tilde{a}$ is close enough to $a$ in $C^{2}([0, T] ; C(\bar{\Omega}))$. We can therefore absorb the $\delta$ term with the l.h.s. lem.

We are ready now to formulate the stability result for the thermoacoustic prob-

Theorem 3.5. Let $K \subset \Omega$ be a compact set. Let $c, \tilde{c} \in C^{k}, f \in H^{k+1}, k>n / 2$, be as in Theorem 3.3 and let the assumptions of that theorem be satisfied, except for (49). Then

$$
\|\tilde{c}-c\|_{L^{2}(K)} \leq C\left\|\partial_{t}^{2}(\tilde{\Lambda}-\Lambda) f\right\|_{L^{2}([0, T] \times \partial \Omega)},
$$

with $C=C\left(C_{1}, f, c\right)$ uniform if $\tilde{c}$ satisfies $\|\tilde{c}\|_{C^{k}} \leq C_{1}, 1 / C_{1} \leq \tilde{c}, k>n / 2$.

Proof. Apply Theorem 3.4 with $a$ and $F$ as in (3). We only need to prove the statement about the uniformity of $C$. That requires us to estimate $\|\tilde{u}\|_{C^{2}([0, T] \times \partial \Omega)}$ in terms of $\tilde{c}$; see (3) and Theorem 3.4

It is straightforward to see that if $c \in C^{k-1}$, then $\mathbf{A}^{k} \mathbf{f} \in \mathcal{H}$ provided that $\mathbf{f} \in H^{k+1} \times H^{k}$. Then $\mathbf{A}^{k} \mathbf{U}(t) \mathbf{f}$ is locally in $H^{k+1} \times H^{k}$, therefore the first component of $\mathbf{U}(t) \mathbf{f}$ is $C^{k+1-n / 2}$ provided that $k+1-n / 2>0$. We have $k+1-n / 2 \geq 2$ when $k-1 \geq n / 2$. Therefore, $\tilde{u} \in C\left([0, T] ; C^{2}\right)$ when $k>n / 2$. The analysis of the rest of the second derivatives of $u$ is similar. 


\section{ACKNOWLEDGMENTS}

The authors thank Linh Nguyen and Peter Kuchment for fruitful discussions on the mathematics of thermoacoustic tomography, and for attracting their attention to reference [11, Theorem 8.2.2]. Thanks are also due to Daniel Tataru for his help to better understand the various unique continuation results. The essential part of this work was done while both authors were visiting the MSRI in Fall, 2010. We also want to thank the referee, whose valuable suggestions significantly helped to improve the exposition.

\section{REFERENCES}

[1] C. Bardos, G. Lebeau, and J. Rauch. Sharp sufficient conditions for the observation, control, and stabilization of waves from the boundary. SIAM J. Control Optim., 30(5):1024-1065, 1992. MR 1178650 (94b:93067)

[2] A. L. Bukhgeim and M. V. Klibanov. Uniqueness in the large of a class of multidimensional inverse problems. Dokl. Akad. Nauk SSSR, 260(2):269-272, 1981. English translation: Soviet Math. Dokl. 24 (1981), no. 2, 244-247 (1982). MR630135 (83b:35157)

[3] D. Finch, S. K. Patch, and Rakesh. Determining a function from its mean values over a family of spheres. SIAM J. Math. Anal., 35(5):1213-1240 (electronic), 2004. MR2050199 (2005b:35290)

[4] D. Finch and Rakesh. Recovering a function from its spherical mean values in two and three dimensions. in: Photoacoustic Imaging and Spectroscopy, CRC Press, 2009.

[5] Y. Hristova. Time reversal in thermoacoustic tomography - an error estimate. Inverse Problems, 25(5):055008, 2009. MR2501026 (2010d:78036)

[6] Y. Hristova, P. Kuchment, and L. Nguyen. Reconstruction and time reversal in thermoacoustic tomography in acoustically homogeneous and inhomogeneous media. Inverse Problems, 24:055006, 2008. MR2438941 (2010c:65162)

[7] O. Y. Imanuvilov and M. Yamamoto. Global Lipschitz stability in an inverse hyperbolic problem by interior observations. Inverse Problems, 17(4):717-728, 2001. Special issue to celebrate Pierre Sabatier's 65th birthday (Montpellier, 2000). MR,1861478 (2002i:35204)

[8] O. Y. Imanuvilov and M. Yamamoto. Global uniqueness and stability in determining coefficients of wave equations. Comm. Partial Differential Equations, 26(7-8):1409-1425, 2001. MR.1855284 (2002j:35310)

[9] O. Y. Imanuvilov and M. Yamamoto. Determination of a coefficient in an acoustic equation with a single measurement. Inverse Problems, 19(1):157-171, 2003. MR.1964256 (2004c:35415)

[10] V. Isakov. Carleman type estimates and their applications. In New analytic and geometric methods in inverse problems, pages 93-125. Springer, Berlin, 2004. MR2053418(2006c:35023)

[11] V. Isakov. Inverse problems for partial differential equations, volume 127 of Applied Mathematical Sciences. Springer, New York, second edition, 2006. MR2193218(2006h:35279)

[12] A. Katchalov, Y. Kurylev, and M. Lassas. Inverse boundary spectral problems, volume 123 of Chapman 8 Hall/CRC Monographs and Surveys in Pure and Applied Mathematics. Chapman \& Hall/CRC, Boca Raton, FL, 2001. MR1889089 (2003e:58045)

[13] P. Kuchment and L. Kunyansky. Mathematics of thermoacoustic tomography. European J. Appl. Math., 19(2):191-224, 2008. MR2400720(2009c:92026)

[14] I. Lasiecka, J.-L. Lions, and R. Triggiani. Nonhomogeneous boundary value problems for second order hyperbolic operators. J. Math. Pures Appl., 65(2):149-192, 1986. MR867669 (88c:35092)

[15] I. Lasiecka, R. Triggiani, and P. F. Yao. Exact controllability for second-order hyperbolic equations with variable coefficient-principal part and first-order terms. In Proceedings of the Second World Congress of Nonlinear Analysts, Part 1 (Athens, 1996), volume 30, pages 111-122, 1997. MR1489773 (99a:35158)

[16] S. K. Patch. Thermoacoustic tomography - consistency conditions and the partial scan problem. Physics in Medicine and Biology, 49(11):2305-2315, 2004.

[17] J.-P. Puel and M. Yamamoto. On a global estimate in a linear inverse hyperbolic problem. Inverse Problems, 12(6):995-1002, 1996. MR1421661(97k:35271) 
[18] J. Qian, P. Stefanov, G. Uhlmann, and H. Zhao. An efficient neumann-series based algorithm for thermoacoustic and photoacoustic tomography with variable sound speed. SIAM J. Imaging Sciences 4 (2011), no. 3, 850-883. MR2836390

[19] V. G. Romanov. Carleman estimates for a second-order hyperbolic equation. Sibirsk. Mat. Zh., 47(1):169-187, 2006. MR2215303 (2006m:35216)

[20] V. A. Sharafutdinov. Integral geometry of tensor fields. Inverse and Ill-posed Problems Series. VSP, Utrecht, 1994. MR1374572 (97h:53077)

[21] P. Stefanov and G. Uhlmann. Stability estimates for the hyperbolic Dirichlet to Neumann map in anisotropic media. J. Funct. Anal., 154(2):330-358, 1998. MR1612709 (99f:35120)

[22] P. Stefanov and G. Uhlmann. Boundary rigidity and stability for generic simple metrics. $J$. Amer. Math. Soc., 18(4):975-1003, 2005. MR2163868(2006h:53031)

[23] P. Stefanov and G. Uhlmann. Thermoacoustic tomography with variable sound speed. Inverse Problems, 25(7):075011, 16, 2009. MR 2519863(2010i:35439)

[24] P. Stefanov and G. Uhlmann. Thermoacoustic tomography arising in brain imaging. Inverse Problems, 27(4):045004, 26, 2011. MR2781028

[25] D. Tataru. On the regularity of boundary traces for the wave equation. Ann. Scuola Norm. Sup. Pisa Cl. Sci. (4), 26(1):185-206, 1998. MR1633000 (99e:35129)

[26] D. Tataru. Unique continuation for operators with partially analytic coefficients. J. Math. Pures Appl. (9), 78(5):505-521, 1999. MR1697040 (2000e:35005)

[27] D. Tataru. Unique continuation problems for partial differential equations. In Geometric methods in inverse problems and PDE control, volume 137 of IMA Vol. Math. Appl., pages 239-255. Springer, New York, 2004. MR2169906

[28] M. E. Taylor. Partial differential equations. I, volume 115 of Applied Mathematical Sciences. Springer-Verlag, New York, 1996. Basic theory. MR1395148 (98b:35002b)

[29] R. Triggiani and P. F. Yao. Carleman estimates with no lower-order terms for general Riemann wave equations. Global uniqueness and observability in one shot. Appl. Math. Optim., 46(23):331-375, 2002. Special issue dedicated to the memory of Jacques-Louis Lions. MR,1944764 (2003j:93042)

[30] P.-F. Yao. On the observability inequalities for exact controllability of wave equations with variable coefficients. SIAM J. Control Optim., 37(5):1568-1599 (electronic), 1999. MR.1710233 (2000m:93027)

Department of Mathematics, Purdue University, West Lafayette, Indiana 47907

Department of Mathematics, University of Washington, Seattle, Washington 98195 